\title{
ON THE cd-INDEX AND $\gamma$-VECTOR OF S*-SHELLABLE CW-SPHERES
}

\author{
SATOSHI MURAI AND ERAN NEVO
}

\begin{abstract}
We show that the $\gamma$-vector of the order complex of any polytope is the $f$-vector of a balanced simplicial complex. This is done by proving this statement for a subclass of Stanley's S-shellable CW-spheres which includes all polytopes. The proof shows that certain parts of the cd-index, when specializing $\mathbf{c}=1$ and considering the resulted polynomial in $\mathbf{d}$, are the $f$-polynomials of simplicial complexes that can be colored with "few" colors. We conjecture that the cd-index of a regular $\mathrm{CW}$-sphere is itself the flag $f$-vector of a colored simplicial complex in a certain sense.
\end{abstract}

\section{INTRODUCTION}

Let $P$ be an $(n-1)$-dimensional regular $\mathrm{CW}$-sphere (that is, a regular $\mathrm{CW}$-complex which is homeomorphic to an $(n-1)$-dimensional sphere). In face enumeration, one of the most important combinatorial invariants of $P$ is the cd-index. The cd-index $\Phi_{P}(\mathbf{c}, \mathbf{d})$ of $P$ is a non-commutative polynomial in the variables $\mathbf{c}$ and $\mathbf{d}$ that encodes the flag $f$-vector of $P$. By the result of Stanley [St1] and Karu [Ka], it is known that the cd-index $\Phi_{P}(\mathbf{c}, \mathbf{d})$ has non-negative integer coefficients. On the other hand, a characterization of the possible cd-indices for regular CW-spheres, or other related families, e.g. Gorenstien* posets, is still beyond reach. In this paper we take a step in this direction and establish some non-trivial upper bounds, as we detail now.

If we substitute 1 for $\mathbf{c}$ in $\Phi_{P}(\mathbf{c}, \mathbf{d})$, we obtain a polynomial of the form

$$
\Phi_{P}(1, \mathbf{d})=\delta_{0}+\delta_{1} \mathbf{d}+\cdots+\delta_{\left\lfloor\frac{n}{2}\right\rfloor} \mathbf{d}^{\left\lfloor\frac{n}{2}\right\rfloor},
$$

where $\left\lfloor\frac{n}{2}\right\rfloor$ is the integer part of $\frac{n}{2}$, such that each $\delta_{i}$ is a non-negative integer. In other words, $\delta_{i}$ is the sum of coefficients of monomials in $\Phi_{P}(\mathbf{c}, \mathbf{d})$ for which $\mathbf{d}$ appears $i$ times.

Let $\Delta$ be a (finite abstract) simplicial complex on the vertex set $V$. We say that $\Delta$ is $k$-colored if there is a map $c: V \rightarrow[k]=\{1,2, \ldots, k\}$, called a $k$-coloring map of $\Delta$, such that if $\{x, y\}$ is an edge of $\Delta$ then $c(x) \neq c(y)$. Let $f_{i}(\Delta)$ denote the number of elements $F \in \Delta$ having cardinality $i+1$, where $f_{-1}(\Delta)=1$. The main result of this paper is the following.

Theorem 1.1. Let $P$ be an $(n-1)$-dimensional $S^{*}$-shellable regular $C W$-sphere, and let $\Phi_{P}(1, \mathbf{d})=\delta_{0}+\delta_{1} \mathbf{d}+\cdots+\delta_{\left\lfloor\frac{n}{2}\right\rfloor} \mathbf{d}^{\left\lfloor\frac{n}{2}\right\rfloor}$. Then there exists an $\left\lfloor\frac{n}{2}\right\rfloor$-colored simplicial complex $\Delta$ such that

$$
\delta_{i}=f_{i-1}(\Delta) \quad \text { for } i=0,1, \ldots,\left\lfloor\frac{n}{2}\right\rfloor .
$$

Key words and phrases. polytope, $\gamma$-vector, $c d$-index, balanced simplicial complex.

Research of the first author was partially supported by KAKENHI 22740018. Research of the second author was partially supported by an NSF Award DMS-0757828. 
The precise definition of $\mathrm{S}^{*}$-shellability is given in Section 2. The most important class of $\mathrm{S}^{*}$-shellable $\mathrm{CW}$-spheres is the class of the boundary complexes of polytopes. By the Kruskal-Katona Theorem (see e.g. [St2, II, Theorem 2.1]), the above theorem gives a certain upper bound on $\delta_{i}$ in terms of $\delta_{i-1}$. Better upper bounds are given by Frankl-Füredi-Kalai theorem which characterizes the $f$-vectors of $k$-colored complexes $[\mathrm{FFK}]$.

The numbers $\delta_{0}, \delta_{1}, \delta_{2}, \ldots$ relate to the $\gamma$-vector (see Section 4 for the definition) of the barycentric subdivision (order complex) of $P$, namely the simplicial complex whose elements are the chains of nonempty cells in $P$ ordered by inclusion. Indeed, as an application of Theorem 1.1 we prove the following.

Theorem 1.2. Let $P$ be an $(n-1)$-dimensional $S^{*}$-shellable regular $C W$-sphere and let $\operatorname{sd}(P)$ be the barycentric subdivision of $P$. Then there exists an $\left\lfloor\frac{n}{2}\right\rfloor$-colored simplicial complex $\Gamma$ such that

$$
\gamma_{i}(\operatorname{sd}(P))=f_{i-1}(\Gamma) \quad \text { for } i=0,1, \ldots,\left\lfloor\frac{n}{2}\right\rfloor .
$$

Recall that an $(n-1)$-dimensional simplicial complex is said to be balanced if it is $n$-colored. If $P$ is the boundary complex of an arbitrary convex $n$-dimensional polytope, then $\delta_{\left\lfloor\frac{n}{2}\right\rfloor}>0$ and we conclude the following.

Corollary 1.3. Let $P$ be the boundary complex of an n-dimensional polytope. Then the $\gamma$-vector of $\operatorname{sd}(P)$ is the $f$-vector of a balanced simplicial complex.

The above corollary supports the conjecture of Nevo and Petersen [NP, Conjecture 6.3] which states that the $\gamma$-vector of a flag homology sphere is the $f$-vector of a balanced simplicial complex. This conjecture was verified for the barycentric subdivision of simplicial homology spheres (in this case all the cells are simplices) in $[\mathrm{NPT}]$.

It would be natural to ask if the above theorems hold for all regular CW-spheres (or more generally, Gorenstein* posets). We conjecture a stronger statement on the cd-index, see Conjecture 4.3.

This paper is organized as follows: in Section 2 we recall some known results on the cd-index and define $\mathrm{S}^{*}$-shellability, in Section 3 we prove our main theorem, Theorem 1.1, in Section 4 we derive consequences for $\gamma$-vectors and present a conjecture on the cd-index, Conjecture 4.3.

\section{2. cd-INDEX OF $\mathrm{S}^{*}$-SHELLABLE CW-SPHERES}

In this section we recall some known results on the cd-index.

Let $P$ be a graded poset of rank $n+1$ with the minimal element $\hat{0}$ and the maximal element $\hat{1}$. Let $\rho$ denote the rank function of $P$. For $S \subset[n]=\{1,2, \ldots, n\}$, a chain $\hat{0}=\sigma_{0}<\sigma_{1}<\sigma_{2}<\cdots<\sigma_{k+1}=\hat{1}$ of $P$ is called an $S$-flag if $\left\{\rho\left(\sigma_{1}\right), \ldots, \rho\left(\sigma_{k}\right)\right\}=S$. Let $f_{S}(P)$ be the number of $S$-flags of $P$. Define $h_{S}(P)$ by

$$
h_{S}(P)=\sum_{T \subset S}(-1)^{|S|-|T|} f_{T}(P),
$$

where $|X|$ denotes the cardinality of a finite set $X$. The vectors $\left(f_{S}(P): S \subset[n]\right)$ and $\left(h_{S}(P): S \subset[n]\right)$ are called the flag $f$-vector and flag $h$-vector of $P$ respectively. 
Now we recall the definition of the cd-index. For $S \subset[n]$, we define a noncommutative monomial $u_{S}=u_{1} u_{2} \cdots u_{n}$ in variables $\mathbf{a}$ and $\mathbf{b}$ by $u_{i}=\mathbf{a}$ if $i \notin S$ and $u_{i}=\mathbf{b}$ if $i \in S$. Let

$$
\Psi_{P}(\mathbf{a}, \mathbf{b})=\sum_{S \subset[n]} h_{S}(P) u_{S}
$$

For a graded poset $P$, let $\operatorname{sd}(P)$ be the order complex of $P-\{\hat{0}, \hat{1}\}$. Thus

$$
\operatorname{sd}(P)=\left\{\left\{\sigma_{1}, \sigma_{2}, \ldots, \sigma_{k}\right\} \subset P-\{\hat{0}, \hat{1}\}: \sigma_{1}<\sigma_{2}<\cdots<\sigma_{k}\right\} .
$$

We say that $P$ is Gorenstein* if the simplicial complex $\operatorname{sd}(P)$ is a homology sphere. It is known that if $P$ is Gorenstein* then $\Psi_{P}(\mathbf{a}, \mathbf{b})$ can be written as a polynomial $\Phi_{P}(\mathbf{c}, \mathbf{d})$ in $\mathbf{c}=\mathbf{a}+\mathbf{b}$ and $\mathbf{d}=\mathbf{a b}+\mathbf{b a}[\mathrm{BK}]$, and this non-commutative polynomial $\Phi_{P}(\mathbf{c}, \mathbf{d})$ is called the $\mathbf{c d}$-index of $P$. Moreover, by the celebrated results due to Stanley [St1] (for convex polytopes) and Karu [Ka] (for Gorenstein* posets), the coefficients of $\Phi_{P}(\mathbf{c}, \mathbf{d})$ are non-negative integers.

Next, we define $\mathrm{S}^{*}$-shellability of regular $\mathrm{CW}$-spheres by slightly modifying the definition of S-shellability introduced by Stanley [St1, Definition 2.1].

Let $P$ be a regular CW-sphere (a regular CW-complex which is homeomorphic to a sphere) and $\mathcal{F}(P)$ its face poset. Then the order complex of $\mathcal{F}(P)$ is a triangulation of a sphere, so the poset $\mathcal{F}(P) \cup\{\hat{0}, \hat{1}\}$ is Gorenstein*. We define the cd-index of $P$ by $\Phi_{P}(\mathbf{c}, \mathbf{d})=\Phi_{\mathcal{F}(P) \cup\{\hat{0}, \hat{1}\}}(\mathbf{c}, \mathbf{d})$. For any cell $\sigma$ of $P$, we write $\bar{\sigma}$ for the closure of $\sigma$. For an $(n-1)$-dimensional regular $\mathrm{CW}$-sphere $P$, let $\Sigma P$ be the suspension of $P$, namely, $\Sigma P$ is the $n$-dimensional regular $\mathrm{CW}$-sphere obtained from $P$ by attaching two $n$-dimensional cells $\tau_{1}$ and $\tau_{2}$ such that $\partial \bar{\tau}_{1}=\partial \bar{\tau}_{2}=P$. Also, for an $(n-1)$ dimensional regular CW-ball $P$ (a regular CW-complex which is homeomorphic to an $(n-1)$-dimensional ball), let $P^{\prime}$ be the $(n-1)$-dimensional regular $C W$-sphere which is obtained from $P$ by adding an $(n-1)$-dimensional cell $\tau$ so that $\partial \bar{\tau}=\partial P$.

Definition 2.1. Let $P$ be an $(n-1)$-dimensional regular $\mathrm{CW}$-sphere. We say that $P$ is $S^{*}$-shellable if either $P=\{\emptyset\}$ or there is an order $\sigma_{1}, \sigma_{2}, \ldots, \sigma_{r}$ of the facets of $P$ such that the following conditions hold.

(a) $\partial \bar{\sigma}_{1}$ is $\mathrm{S}^{*}$-shellable.

(b) For $1 \leq i \leq r-1$, let

$$
\Omega_{i}=\bar{\sigma}_{1} \cup \bar{\sigma}_{2} \cup \cdots \cup \bar{\sigma}_{i}
$$

and for $2 \leq i \leq r-1$ let

$$
\Gamma_{i}=\overline{\left[\partial \bar{\sigma}_{i} \backslash\left(\partial \bar{\sigma}_{i} \cap \Omega_{i-1}\right)\right]}
$$

Then both $\Omega_{i}$ and $\Gamma_{i}$ are regular CW-balls of dimension $(n-1)$ and $(n-2)$ respectively, and $\Gamma_{i}^{\prime}$ is $\mathrm{S}^{*}$-shellable with the first facet of the shelling being the facet which is not in $\Gamma_{i}$.

Remark 2.2. The difference between the above definition and Stanley's S-shellability is that S-shellability only assume that $P$ and $\Gamma_{i}^{\prime}$ are Eulerian and assume no conditions on $\Omega_{i}$. However, $\mathrm{S}^{*}$-shellable regular $\mathrm{CW}$-spheres are S-shellable, and the boundary complex of convex polytopes are $S^{*}$-shellable by the line shelling [BM]. We leave the verification of this fact to the readers.

The next recursive formula is due to Stanley [St1]. 
Lemma 2.3 (Stanley). With the same notation as in Definition 2.1, for $i=$ $1,2, \ldots, r-2$, one has

$$
\Phi_{\Omega_{i+1}^{\prime}}(\mathbf{c}, \mathbf{d})=\Phi_{\Omega_{i}^{\prime}}(\mathbf{c}, \mathbf{d})+\left\{\Phi_{\Gamma_{i+1}^{\prime}}(\mathbf{c}, \mathbf{d})-\Phi_{\Sigma\left(\partial \Gamma_{i+1}\right)}(\mathbf{c}, \mathbf{d})\right\} \mathbf{c}+\Phi_{\partial \Gamma_{i+1}}(\mathbf{c}, \mathbf{d}) \mathbf{d} .
$$

Since $\Omega_{r-1}^{\prime}=P$ the above formula gives a way to compute the cd-index of $P$ recursively.

Next, we recall a result of Ehrenborg and Karu proving that the cd-index increases by taking subdivisions. Let $P$ and $Q$ be regular CW-complexes, and let $\phi: \mathcal{F}(P) \rightarrow$ $\mathcal{F}(Q)$ be a poset map. For a subcomplex $Q^{\prime}=\sigma_{1} \cup \cdots \cup \sigma_{s} \subset Q$, where each $\sigma_{i}$ is a cell of $Q$, we write $\phi^{-1}\left(Q^{\prime}\right)=\phi^{-1}\left(\sigma_{1}\right) \cup \cdots \cup \phi^{-1}\left(\sigma_{s}\right)$.

Following [EK, Definition 2.6], for $(n-1)$-dimensional regular $\mathrm{CW}$-spheres $P$ and $\hat{P}$, we say that $\hat{P}$ is a subdivision of $P$ if there is an order preserving surjective poset map $\phi: \mathcal{F}(\hat{P}) \rightarrow \mathcal{F}(P)$, satisfying that for any cell $\sigma$ of $P, \phi^{-1}(\bar{\sigma})$ is a homology ball having the same dimension as $\sigma$ and $\phi^{-1}(\partial \bar{\sigma})=\partial\left(\phi^{-1}(\bar{\sigma})\right)$.

The following result was proved in [EK, Theorem 1.5].

Lemma 2.4 (Ehrenborg-Karu). Let $P$ and $\hat{P}$ be $(n-1)$-dimensional regular $C W$ spheres. If $\hat{P}$ is a subdivision of $P$ then one has a coefficientwise inequality $\Phi_{\hat{P}}(\mathbf{c}, \mathbf{d}) \geq$ $\Phi_{P}(\mathbf{c}, \mathbf{d})$

Back to $\mathrm{S}^{*}$-shellable regular CW-spheres, with the same notation as in Definition 2.1, $\Omega_{i}^{\prime}$ is a subdivision of $\Sigma\left(\partial \Omega_{i}\right)$ and $\partial \Omega_{i}$ is a subdivision of $\Sigma\left(\partial \Gamma_{i+1}\right)$. Indeed, for the first statement, if $\tau_{1}$ and $\tau_{2}$ are the facets of $\Sigma\left(\partial \Omega_{i}\right)$ then define $\phi: \mathcal{F}\left(\Omega_{i}^{\prime}\right) \rightarrow$ $\mathcal{F}\left(\Sigma\left(\partial \Omega_{i}\right)\right)$ by

$$
\phi(\sigma)= \begin{cases}\sigma, & \text { if } \sigma \in \partial \Omega_{i}, \\ \tau_{1}, & \text { if } \sigma \text { is an interior face of } \Omega_{i}, \\ \tau_{2}, & \text { if } \sigma \notin \Omega_{i} .\end{cases}
$$

Similarly, for the second statement, if $\tau_{1}$ and $\tau_{2}$ are the facets of $\Sigma\left(\partial \Gamma_{i+1}\right)$ then define $\phi: \mathcal{F}\left(\partial \Omega_{i}\right) \rightarrow \mathcal{F}\left(\Sigma\left(\partial \Gamma_{i+1}\right)\right)$ by

$$
\phi(\sigma)= \begin{cases}\sigma, & \text { if } \sigma \in \partial \Gamma_{i+1}, \\ \tau_{1}, & \text { if } \sigma \in \bar{\sigma}_{i+1} \backslash \partial \Gamma_{i+1}, \\ \tau_{2}, & \text { otherwise. }\end{cases}
$$

Since $\Phi_{\Sigma P}(\mathbf{c}, \mathbf{d})=\Phi_{P}(\mathbf{c}, \mathbf{d}) \mathbf{c}$ for any regular CW-sphere $P$ (see [St1, Lemma 1.1]), Lemma 2.4 shows

Lemma 2.5. With the same notation as in Definition 2.1, for $i=2,3, \ldots, r-2$, one has $\Phi_{\Omega_{i}^{\prime}}(\mathbf{c}, \mathbf{d}) \geq \Phi_{\partial \Gamma_{i+1}}(\mathbf{c}, \mathbf{d}) \mathbf{c}^{2}$.

\section{Proof of the MAIN THEOREM}

In this section, we prove Theorem 1.1.

For a homogeneous cd-polynomial $\Phi$ (i.e., a homogeneous polynomial of $\mathbb{Z}\langle\mathbf{c}, \mathbf{d}\rangle$ with $\operatorname{deg} \mathbf{c}=1$ and $\operatorname{deg} \mathbf{d}=2$ ) of degree $n$, we define $\Phi_{0}, \Phi_{2}, \ldots, \Phi_{n}$ by

$$
\Phi=\Phi_{0}+\Phi_{2} \mathbf{d} \mathbf{c}^{n-2}+\Phi_{3} \mathbf{d} \mathbf{c}^{n-3}+\cdots+\Phi_{n-1} \mathbf{d} \mathbf{c}+\Phi_{n} \mathbf{d}
$$

where $\Phi_{0}=\alpha \mathbf{c}^{n}$ for some $\alpha \in \mathbb{Z}$ and each $\Phi_{k}$ is a cd-polynomial of degree $k-2$ for $k \geq 2$. Also, we write $\Phi_{\leq k}=\Phi_{0}+\Phi_{2} \mathbf{d c}^{n-2}+\cdots+\Phi_{k} \mathbf{d} \mathbf{c}^{n-k}$. 


\section{Definition 3.1.}

- A vector $\left(\delta_{0}, \delta_{1}, \ldots, \delta_{s}\right) \in \mathbb{Z}^{s+1}$ is said to be $k$-FFK if there is a $k$-colored simplicial complex $\Delta$ such that $\delta_{i}=f_{i-1}(\Delta)$ for $i=0,1, \ldots, s$. $(\{\emptyset\}$ is a 0-colored simplicial complex.) A homogeneous cd-polynomial $\Phi=\Phi(\mathbf{c}, \mathbf{d})$ is said to be $k$-FFK if, when we write $\Phi(1, \mathbf{d})=\delta_{0}+\delta_{1} \mathbf{d}+\cdots+\delta_{s} \mathbf{d}^{s}$, the vector $\left(\delta_{0}, \delta_{1}, \ldots, \delta_{s}\right)$ is $k$-FFK.

- A homogeneous cd-polynomial $\Phi$ of degree $n$ is said to be primitive if the coefficient of $\mathbf{c}^{n}$ in $\Phi$ is 1 .

- Let $\Phi$ be a homogeneous cd-polynomial. A primitive homogeneous cdpolynomial $\Psi$ is said to be $k$-good for $\Phi$ if $\Psi$ is $k$-FFK and $\Phi(1, \mathbf{d}) \geq \Psi(1, \mathbf{d})$. Also, we say that a homogeneous cd-polynomial $\Psi$ is $k$-good for $\Phi$ if it is the sum of primitive homogeneous cd-polynomials that are $k$-good for $\Phi$.

Next, we recall Frankl-Füredi-Kalai theorem [FFK], which characterizes all possible $f$-vectors of colored complexes. Let $\mathbb{N}_{i}^{(k)}=\left\{i+j k: j \in \mathbb{Z}_{\geq 0}\right\}$ for $i=1,2, \ldots, k$ and

$$
\mathcal{C}^{(k)}=\left\{F \subset \mathbb{N}:\left|F \cap \mathbb{N}_{i}^{(k)}\right| \leq 1 \text { for } i=1,2, \ldots, k\right\},
$$

where $\mathbb{N}$ is the set of positive integers. Let $>_{\text {rev }}$ be the reverse lexicographic order induced by $1>_{\text {rev }} 2>_{\text {rev }} \cdots$. Thus, for finite subsets $F \subset \mathbb{N}$ and $G \subset \mathbb{N}$ with $|F|=|G|$, one has $F>_{\text {rev }} G$ if the largest integer in the symmetric difference $(F \backslash G) \cup(G \backslash F)$ is contained in $G$. A $k$-colored compressed complex is a simplicial complex $\Delta$ such that $\Delta \subset \mathcal{C}^{(k)}$ and that, for every $F \in \Delta$ and $G \in \mathcal{C}^{(k)}$ with $|G|=|F|$ and $G>_{\text {rev }} F$, one has $G \in \Delta$. Since $>_{\text {rev }}$ is a total order on the set of finite subsets of $\mathbb{N}$ having the same cardinality, $k$-colored compressed complex is uniquely determined by its $f$-vector.

Theorem 3.2 (Frankl-Füredi-Kalai). A vector $\left(\delta_{0}, \delta_{1}, \ldots, \delta_{s}\right) \in \mathbb{Z}^{s+1}$ is $k$-FFK if and only if there is a $k$-colored compressed complex $\Delta$ such that $f_{i-1}(\Delta)=\delta_{i}$ for $i=0,1, \ldots, s$.

We will use the following observation, which follows from [NPT, Lemma 3.1]:

Lemma 3.3. If $\Phi$ is a k-FFK homogeneous cd-polynomial of degree $n$, and if $\Psi^{\prime}$ and $\Psi^{\prime \prime}$ are homogeneous cd-polynomials of degree $n^{\prime}$ and $n^{\prime \prime}$ respectively, where $n^{\prime}, n^{\prime \prime} \leq n-2$, which are $k$-good for $\Phi$ then

$$
\Phi+\Psi^{\prime} \mathbf{d} \mathbf{c}^{n-n^{\prime}-2} \text { and } \Phi+\Psi^{\prime} \mathbf{d} \mathbf{c}^{n-n^{\prime}-2}+\Psi^{\prime \prime} \mathbf{d} \mathbf{c}^{n-n^{\prime \prime}-2}
$$

are $(k+1)-F F K$.

Proof. For a simplicial complex $\Gamma$, we write $f(\Gamma, \mathbf{d})=1+f_{0}(\Gamma) \mathbf{d}+f_{1}(\Gamma) \mathbf{d}^{2}+\cdots$. There are $k$-colored complexes $\Delta, \Delta^{(1)}, \cdots, \Delta^{(m)}, \cdots, \Delta^{(s)}$ such that $f(\Delta, \mathbf{d})=$ $\Phi(1, \mathbf{d}), \sum_{1 \leq i \leq m} f\left(\Delta^{(i)}, \mathbf{d}\right)=\Psi^{\prime}(1, \mathbf{d}), \sum_{m+1 \leq i \leq s} f\left(\Delta^{(i)}, \mathbf{d}\right)=\Psi^{\prime \prime}(1, \mathbf{d})$ and $\Phi(1, d) \geq$ $f\left(\Delta^{(i)}, d\right)$ for all $1 \leq i \leq s$. By Frankl-Füredi-Kalai theorem, we may assume that all these complexes are $k$-colored compressed. Then, since $\Phi(1, \mathbf{d}) \geq \Psi^{\prime}(1, \mathbf{d})$ and $\Phi(1, \mathbf{d}) \geq \Psi^{\prime \prime}(1, \mathbf{d})$, each $\Delta^{(i)}$ is a subcomplex of $\Delta$. For $i=1,2, \ldots, s$, let

$$
\Gamma^{(i)}=\Delta \bigcup\left\{\bigcup_{j=1}^{i}\left\{F \cup\left\{v_{j}\right\}: F \in \Delta^{(j)}\right\}\right\},
$$


where $v_{1}, \ldots, v_{s}$ are new vertices. Since each $\Delta^{(j)}$ is a subcomplex of $\Delta, \Gamma^{(i)}$ is a simplicial complex. Also, $f\left(\Gamma^{(m)}, \mathbf{d}\right)=\left(\Phi+\Psi^{\prime} \mathbf{d} \mathbf{c}^{n-n^{\prime}-2}\right)(1, \mathbf{d})$ and $f\left(\Gamma^{(s)}, \mathbf{d}\right)=$ $\left(\Phi+\Psi^{\prime} \mathbf{d} \mathbf{c}^{n-n^{\prime}-2}+\Psi^{\prime \prime} \mathbf{d} \mathbf{c}^{n-n^{\prime \prime}-2}\right)(1, \mathbf{d})$. We claim that each $\Gamma^{(i)}$ is $(k+1)$-colored. Let $V$ be the vertex set of $\Delta$ and $c: V \rightarrow[k]$ a $k$-coloring map of $\Delta$. Then the map $\hat{c}: V \cup\left\{v_{1}, \ldots, v_{i}\right\} \rightarrow[k+1]$ defined by $\hat{c}(x)=c(x)$ if $x \in V$ and $\hat{c}(x)=k+1$ if $x \notin V$ is a $(k+1)$-coloring map of $\Gamma^{(i)}$.

Let $P$ be an $(n-1)$-dimensional $S^{*}$-shellable regular $\mathrm{CW}$-sphere with the shelling $\sigma_{1}, \ldots, \sigma_{r}$. Keeping the notation in Definition 2.1, to simplify notations, we use the following symbols.

$$
\begin{aligned}
\Phi^{(i)} & =\Phi^{(i)}(\mathbf{c}, \mathbf{d})=\Phi_{\Omega_{i}^{\prime}}(\mathbf{c}, \mathbf{d}) \\
\Phi & =\Phi_{P}(\mathbf{c}, \mathbf{d})=\Phi^{(r-1)} \\
\Psi^{(i)} & =\Phi_{\Gamma_{i+1}^{\prime}}(\mathbf{c}, \mathbf{d})-\Phi_{\Sigma\left(\partial \Gamma_{i+1}\right)}(\mathbf{c}, \mathbf{d}) \\
\Psi & =\sum_{i=1}^{r-2} \Psi^{(i)} \\
\Pi & =\Phi-\Phi^{(1)} .
\end{aligned}
$$

Thus Stanley's recursive formula, Lemma 2.3, says

$$
\Phi^{(i+1)}=\Phi^{(i)}+\Psi^{(i)} \mathbf{c}+\Phi_{\partial \Gamma_{i+1}}(\mathbf{c}, \mathbf{d}) \mathbf{d}
$$

and

$$
\Pi=\Psi \mathbf{c}+\sum_{i=1}^{r-2} \Phi_{\partial \Gamma_{i+1}}(\mathbf{c}, \mathbf{d}) \mathbf{d} .
$$

The last part of the following proposition is a restatement of Theorem 1.1.

Proposition 3.4. With notation as above, the following holds.

(1) For $2 \leq k \leq n, \Psi_{k}^{(i)}$ is $\left\lfloor\frac{k}{2}-1\right\rfloor$-good for $\Phi_{\leq k-2}^{(i)}+\Psi_{\leq k-2}^{(i)} \mathbf{c}$.

(2) For $2 \leq k \leq n$, $\Pi_{k}$ is $\left\lfloor\frac{k}{2}-1\right\rfloor$-good for $\Phi_{\leq k-2}^{(1)}+\Pi_{\leq k-2}$.

(3) For $2 \leq k \leq n, \Phi_{k}$ is $\left\lfloor\frac{k}{2}-1\right\rfloor$-good for $\Phi_{\leq k-2}$.

(4) For $0 \leq k \leq n, \Phi_{\leq k}$ is $\left\lfloor\frac{k}{2}\right\rfloor-F F K$. In particular, the cd-index of $P$ is $\left\lfloor\frac{n}{2}\right\rfloor-$ FFK.

Proof. The proof is by induction on dimension, where all statements clearly hold for $n=0,1$. Suppose that all statements are true up to dimension $n-2$. To simplify notations, for a regular CW-sphere $Q$, we write $\Phi_{Q}=\Phi_{Q}(\mathbf{c}, \mathbf{d})$.

Proof of (1). By applying the induction hypothesis to $\Gamma_{i+1}^{\prime}$ (use statement(2)), each $\Psi_{k}^{(i)}$ is $\left\lfloor\frac{k}{2}-1\right\rfloor$-good for $\left(\Phi_{\Sigma\left(\partial \Gamma_{i+1}\right)}\right)_{\leq k-2}^{(i)}+\Psi_{\leq k-2}^{(i)}$. Thus, $\Psi_{k}^{(i)}$ is $\left\lfloor\frac{k}{2}-1\right\rfloor$-good for $\left(\Phi_{\Sigma\left(\partial \Gamma_{i+1}\right)}\right)_{\leq k-2}^{(i)} \mathbf{c}+\Psi_{\leq k-2}^{(i)} \mathbf{c}$. By Lemma 2.5,

$$
\Phi_{\Sigma\left(\partial \Gamma_{i+1}\right)} \mathbf{c}=\Phi_{\partial \Gamma_{i+1}} \mathbf{c}^{2} \leq \Phi_{\Omega_{i}^{\prime}}=\Phi^{(i)} .
$$

Since $(\Upsilon \mathbf{c})_{j}=\Upsilon_{j}$ for any homogeneous cd-polynomial $\Upsilon, \Psi_{k}^{(i)}$ is $\left\lfloor\frac{k}{2}-1\right\rfloor$-good for $\Phi_{\leq k-2}^{(i)}+\Psi_{\leq k-2}^{(i)} \mathbf{c}$. 
Proof of (2). By the definition of $\Pi$,

$$
\Pi_{k}=\sum_{i=1}^{r-2} \Psi_{k}^{(i)} \text { for } k<n
$$

and

$$
\Pi_{n}=\sum_{i=1}^{r-2} \Phi_{\partial \Gamma_{i+1}} .
$$

By (1), each $\Psi_{k}^{(i)}$ is $\left\lfloor\frac{k}{2}-1\right\rfloor \operatorname{good}$ for $\Phi_{\leq k-2}^{(i)}+\Psi_{\leq k-2}^{(i)} \mathbf{c}$. Then since

$$
\Phi_{\leq k-2}^{(i)}+\Psi_{\leq k-2}^{(i)} \mathbf{c} \leq \Phi_{\leq k-2}=\Phi_{\leq k-2}^{(1)}+\Pi_{\leq k-2},
$$

$\Pi_{k}$ is $\left\lfloor\frac{k}{2}-1\right\rfloor$-good for $\Phi_{\leq k-2}^{(1)}+\Pi_{\leq k-2}$ for $k<n$. Also, each $\Phi_{\partial \Gamma_{i+1}}$ is $\left\lfloor\frac{n}{2}-1\right\rfloor$-FFK by the induction hypothesis (use (4)), and $\Phi_{\partial \Gamma_{i+1}} \mathbf{c}^{2} \leq \Phi^{(i)}$ by Lemma 2.5. The latter condition clearly says

$$
\Phi_{\partial \Gamma_{i+1}} \mathbf{c}^{2} \leq \Phi_{\leq n-2}^{(i)} \leq \Phi_{\leq n-2}=\Phi_{\leq n-2}^{(1)}+\Pi_{\leq n-2} .
$$

Hence $\Pi_{n}$ is $\left\lfloor\frac{n}{2}-1\right\rfloor$ good for $\Phi_{\leq n-2}^{(1)}+\Pi_{\leq n-2}$.

Proof of (3). Observe that since $\Phi^{(1)}=\Phi_{\partial \bar{\sigma}_{1}} \mathbf{c}$,

$$
\Phi_{k}=\Phi_{k}^{(1)}+\Psi_{k} \text { for } k<n
$$

and

$$
\Phi_{n}=\Pi_{n} .
$$

We already proved that $\Phi_{n}=\Pi_{n}$ is $\left\lfloor\frac{n}{2}-1\right\rfloor$-good for $\Phi_{\leq n-2}$ in the proof of (2). Suppose $k<n$. Since $\Phi^{(1)}=\Phi_{\partial \bar{\sigma}_{1}} \mathbf{c}$, by the induction hypothesis (use (3)), $\Phi_{k}^{(1)}$ is $\left\lfloor\frac{k}{2}-1\right\rfloor$ good for $\Phi_{\leq k-2}^{(1)}$. Since $\Phi_{\leq k-2}^{(1)} \leq \Phi_{\leq k-2}$ and since we already proved that $\Psi_{k}=\Pi_{k}$ is $\left\lfloor\frac{k}{2}-1\right\rfloor$-good for $\Phi_{\leq k-2}$ in the proof of (2), $\Phi_{k}$ is $\left\lfloor\frac{k}{2}-1\right\rfloor$-good for $\Phi_{\leq k-2}$.

Proof of (4). This statement easily follows from (3). For $k=0,1$, the statement is obvious (as $\Phi_{\leq 0}=\Phi_{\leq 1}=\mathbf{c}^{n}$ ). Suppose that $\Phi_{\leq 2 m+1}$ is $m$-FFK, where $m \in \mathbb{Z}_{\geq 0}$. Then both $\Phi_{2 m+2}$ and $\Phi_{2 m+3}$ are $m$-good for $\Phi_{\leq 2 m+1}$ by (3), and therefore $\Phi_{\leq 2 m+2}$ and $\Phi_{\leq 2 m+3}$ are $(m+1)$-FFK by Lemma 3.3.

\section{4. $\gamma$-VECTORS OF POLYTOPES AND A CONJECTURE ON THE cd-INDEX}

$\gamma$-vectors and the cd-index. Let $\Delta$ be an $(n-1)$-dimensional simplicial complex. Then the $h$-vector $h(\Delta)=\left(h_{0}, h_{1}, \ldots, h_{n}\right)$ of $\Delta$ is defined by the relation

$$
\sum_{i=0}^{n} h_{i} x^{n-i}=\sum_{i=0}^{n} f_{i-1}(\Delta)(x-1)^{n-i} .
$$

If $\Delta$ is a simplicial sphere (that is, a triangulation of a sphere), or more generally a homology sphere, then $h_{i}=h_{n-i}$ for all $i$ by the Dehn-Sommerville equations, and in this case the $\gamma$-vector $\left(\gamma_{0}, \gamma_{1}, \ldots, \gamma_{\left\lfloor\frac{n}{2}\right\rfloor}\right)$ of $\Delta$ is defined by the relation

$$
\sum_{i=0}^{n} h_{i} x^{i}=\sum_{i=0}^{\left\lfloor\frac{n}{2}\right\rfloor} \gamma_{i} x^{i}(1+x)^{n-2 i} .
$$


It was conjectured by $\mathrm{Gal}[\mathrm{Ga}]$ that if $\Delta$ is a flag homology sphere then its $\gamma$-vector is non-negative. Recently Nevo and Peterson [NP] further conjectured that the $\gamma$-vector of a flag homology sphere is the $f$-vector of a balanced simplicial complex. These conjectures are open in general, the latter conjecture was verified for barycentric subdivisions of simplicial homology spheres [NPT], and Gal's conjecture is known to be true for barycentric subdivisions of regular CW-spheres by the following fact, combined with Karu's result on the nonnegativity of the cd-index for Gorenstien* posets:

Let $P$ be an $(n-1)$-dimensional regular CW-sphere. The barycentric subdivision $\operatorname{sd}(P)$ of $P$ is the order complex of $\mathcal{F}(P)$. Let $\left(h_{0}, h_{1}, \ldots, h_{n}\right)$ and $\left(\gamma_{0}, \gamma_{1}, \ldots, \gamma_{\left\lfloor\frac{n}{2}\right\rfloor}\right)$ be the $h$-vector and $\gamma$-vector of $\operatorname{sd}(P)$, respectively. Then it is easy to see that $h_{i}=\sum_{S \subset[n],|S|=i} h_{S}(P)$. Thus if $\Phi_{P}(1, \mathbf{d})=\delta_{0}+\delta_{1} \mathbf{d}+\delta_{2} \mathbf{d}^{2}+\cdots+\delta_{\left\lfloor\frac{n}{2}\right\rfloor} \mathbf{d}^{\left\lfloor\frac{n}{2}\right\rfloor}$, then for all $i \geq 0$,

$$
\gamma_{i}=2^{i} \delta_{i}
$$

Since $\delta_{i}$ is non-negative, we conclude that $\gamma_{i}$ is also non-negative.

The next simple statement, combined with Theorem 1.1, proves Theorem 1.2.

Lemma 4.1. With the same notation as above, if $\left(\delta_{0}, \delta_{1}, \ldots, \delta_{\left\lfloor\frac{n}{2}\right\rfloor}\right)$ is $k$-FFK then $\left(\gamma_{0}, \gamma_{1}, \ldots, \gamma_{\left\lfloor\frac{n}{2}\right\rfloor}\right)$ is also $k-F F K$.

Proof. Let $\Delta$ be a $k$-colored simplicial complex on the vertex set $V$ with $f_{i-1}(\Delta)=\delta_{i}$ for all $i \geq 0$ and let $c: V \rightarrow[k]$ be a $k$-coloring map of $\Delta$. Consider a collection of subsets of $W=\left\{x_{v}: v \in V\right\} \cup\left\{y_{v}: v \in V\right\}$

$$
\hat{\Delta}=\left\{x_{G} \cup y_{F \backslash G}: F \in \Delta, G \subset F\right\},
$$

where $x_{H}=\left\{x_{v}: v \in H\right\}$ and $y_{H}=\left\{y_{v}: v \in H\right\}$ for any $H \subset V$. Then $\hat{\Delta}$ is a simplicial complex with $f_{i-1}(\hat{\Delta})=2^{i} f_{i-1}(\Delta)=\gamma_{i}$ for all $i$. The map $\hat{c}: W \rightarrow[k]$, $\hat{c}\left(x_{v}\right)=\hat{c}\left(y_{v}\right)=c(v)$, shows that $\hat{\Delta}$ is $k$-colored.

Proof of Corollary 1.3. By Theorem 1.2, in order to prove Corollary 1.3 it is enough to show that $\delta_{\left\lfloor\frac{n}{2}\right\rfloor}(P)>0$ where $P$ is the boundary complex of an $n$-polytope. Billera and Ehrenborg showed that the cd-index of $n$-polytopes is minimized (coefficientwise) by the $n$-simplex, denoted $\sigma^{n}$ [BE]. Thus, it is enough to verify that $\delta_{\left\lfloor\frac{n}{2}\right\rfloor}\left(\sigma^{n}\right)>0$. It is known that all the cd-coefficients of $\sigma^{n}$ are positive (e.g., by using the Ehrenborg-Readdy formula for the cd-index of a pyramid over a polytope [ER, Theorem 5.2]).

A conjecture on the cd-index. It would be natural to ask if Theorems 1.1 and 1.2 hold for all regular CW-spheres (or all Gorenstein* posets). We phrase a conjecture on the cd-index, that, if true, immediately implies Theorem 1.1, as well as the entire Proposition 3.4(4).

For an arbitrary cd-monomial $w=\mathbf{c}^{s_{0}} \mathbf{d} \mathbf{c}^{s_{1}} \mathbf{d} \cdots \mathbf{d} \mathbf{c}^{s_{k}}$ of degree $n$ (where $0 \leq s_{i}$ for all $i$ and $s_{0}+\cdots+s_{k}+2 k=n$ ), let $F_{w}$ be the following subset of $[n-1]$ :

$$
F_{w}=\left\{s_{0}+1, s_{0}+s_{1}+3, s_{0}+s_{1}+s_{2}+5, \ldots, s_{0}+\cdots+s_{k-1}+2 k-1\right\} .
$$

Note that $F_{w}$ contains no two consecutive numbers. For example, $F_{\mathbf{c}^{n}}=\emptyset, F_{\mathbf{d}^{k}}=$ $\{1,3, \ldots, 2 k-1\}$ and $F_{\mathbf{c d}^{k}}=\{2,4, \ldots, 2 k\}$. Let $\mathcal{A}$ be the set of subsets of $[n-1]$ that have no two consecutive numbers, and let $\mathcal{B}$ be the set of $\mathbf{c d}$-monomials of degree $n$. 
Then $w \mapsto F_{w}$ is a bijection from $\mathcal{B}$ to $\mathcal{A}\left(\right.$ as $k=\left|F_{w}\right|$ and $s_{k}=n-2 k-s_{k-1}-\cdots-s_{0}$ we see that the inverse map exists).

Let $\Delta$ be a $k$-colored simplicial complex with the vertex set $V$ and a $k$-coloring $\operatorname{map} c: V \rightarrow[k]$. For any subset $S \subset[k]$, let $f_{S}(\Delta)=|\{F \in \Delta: c(F)=S\}|$. The vector $\left(f_{S}(\Delta): S \subset[k]\right)$ is called the flag $f$-vector of $\Delta$. Note that the flag $f$-vector of a poset $P$ is equal to the flag $f$-vector of $\operatorname{sd}(P)$ by the coloring map defined by the rank function.

Definition 4.2. Let $\Phi=\sum_{w} a_{w} w$ be a homogeneous cd-polynomial of degree $n$ with $w$ the cd-monomials and $a_{w} \in \mathbb{Z}$. For $S \subset[n-1]$, we define

$$
\alpha_{S}(\Phi)= \begin{cases}a_{w}, & \text { if } S=F_{w} \text { for some } w \in \mathcal{B} \\ 0, & \text { if } S \notin \mathcal{A} .\end{cases}
$$

Conjecture 4.3. Let $P$ be an $(n-1)$-dimensional regular CW-sphere (or more generally, Gorenstein* poset of rank $n+1)$. Then there exists an $(n-1)$-colored simplicial complex $\Delta$ such that $f_{S}(\Delta)=\alpha_{S}\left(\Phi_{P}\right)$ for all $S \subset[n-1]$.

Thus the above conjecture states that the cd-index is itself the flag $f$-vector of a colored complex. If the above conjecture is true then $\Phi_{P}(1, \mathbf{d})=1+f_{0}(\Delta) \mathbf{d}+$ $\cdots+f_{\left\lfloor\frac{n}{2}\right\rfloor-1}(\Delta) \mathbf{d}^{\left\lfloor\frac{n}{2}\right\rfloor}$. Although $\Delta$ is $(n-1)$-colored, this fact implies Theorem 1.1. Indeed, since $f_{S}(\Delta)=\alpha_{S}\left(\Phi_{P}\right)=0$ if $S$ has consecutive numbers, if $c: V \rightarrow[n-1]$ is an $(n-1)$-coloring map of $\Delta$ then the map $\hat{c}: V \rightarrow\left[\left\lfloor\frac{n}{2}\right\rfloor\right]$ defined by $\hat{c}(v)=\left\lfloor\frac{c(v)+1}{2}\right\rfloor$ is an $\left\lfloor\frac{n}{2}\right\rfloor$-coloring map of $\Delta$.

The next result supports the conjecture in low dimension.

Proposition 4.4. Let $P$ be a Gorenstein* poset of rank $n+1$. For all $i, j \in[n-1]$,

$$
\alpha_{\{i\}}\left(\Phi_{P}\right) \alpha_{\{j\}}\left(\Phi_{P}\right) \geq \alpha_{\{i, j\}}\left(\Phi_{P}\right) .
$$

Proof. Let $\left(h_{S}(P): S \subset[n]\right)$ be the flag $h$-vector of $P$. Let $\{i, i+j\} \subset[n-1]$ with $j \geq 2$. What we must prove is $\alpha_{\{i\}}\left(\Phi_{P}\right) \alpha_{\{i+j\}}\left(\Phi_{P}\right) \geq \alpha_{\{i, i+j\}}\left(\Phi_{P}\right)$.

Observe that

$$
\begin{aligned}
h_{[i] \cup\{i+j+1, \ldots, n\}}(P) & =\alpha_{\{i, i+j\}}\left(\Phi_{P}\right)+\alpha_{\{i\}}\left(\Phi_{P}\right)+\alpha_{\{i+j\}}\left(\Phi_{P}\right)+\alpha_{\emptyset}\left(\Phi_{P}\right), \\
h_{[i]}(P) & =\alpha_{\{i\}}\left(\Phi_{P}\right)+\alpha_{\emptyset}\left(\Phi_{P}\right), \\
h_{\{i+j+1, \ldots, n\}}(P) & =\alpha_{\{i+j\}}\left(\Phi_{P}\right)+\alpha_{\emptyset}\left(\Phi_{P}\right)
\end{aligned}
$$

(as $h_{[i] \cup\{i+j+1, \ldots, n\}}(P)$ is the coefficient of $\mathbf{b}^{i} \mathbf{a}^{j} \mathbf{b}^{n-i-j}$ in $\Psi_{P}(\mathbf{a}, \mathbf{b})$, etc.). Since $\alpha_{\emptyset}=1$, it is enough to prove that

$$
h_{[i]}(P) h_{\{i+j+1, \ldots, n\}}(P) \geq h_{[i] \cup\{i+j+1, \ldots, n\}}(P) .
$$

It follows from [St2, III, Theorem 4.6] that there is an $n$-colored simplicial complex $\Delta$ with a coloring map $c: V \rightarrow[n]$ such that $f_{S}(\Delta)=h_{S}(P)$ for all $S \subset[n]$, where $V$ is the vertex set of $\Delta$. Let

$$
\Delta_{S}=\{F \in \Delta: c(F)=S\}
$$

for $S \subset[n]$. Then it is clear that

$$
\Delta_{[i] \cup\{i+j+1, \ldots, n\}} \subset\left\{F \cup G: F \in \Delta_{[i]}, G \in \Delta_{\{i+j+1, \ldots, n\}}\right\},
$$

which implies the desired inequality.

It is straightforward that the above proposition proves the next statement. 
Corollary 4.5. Conjecture 4.3 holds for $n \leq 5$.

Non-existence of d-polynomials. For a Gorenstein* poset $P$, we call $\Phi_{P}(1, \mathbf{d})$ the $\mathbf{d}$-polynomial of $P$. It is a challenging problem to classify all possible $\mathbf{d}$-polynomials of Gorenstein* posets, which give a complete characterization of all possible face vectors of Gorenstein* order complexes since knowing $\mathbf{d}$-polynomials is equivalent to knowing $\gamma$-vectors. The problem is open even for the 3 -dimensional case. To study this problem, by virtue of Theorem 1.1, it is natural to ask which FFK vector is realizable as the $\mathbf{d}$-polynomial of a Gorenstein* poset. We show that not all $\left\lfloor\frac{n}{2}\right\rfloor$-FFK vectors are realizable as the $\mathbf{d}$-polynomial of a Gorenstein* poset of rank $n+1$.

First recall that the ordinal sum $Q_{1}+Q_{2}$ of two disjoint posets $Q_{1}$ and $Q_{2}$ is the poset whose elements are the union of elements in $Q_{1}$ and $Q_{2}$ and whose relations are those in $Q_{1}$ union those in $Q_{2}$ union all $q_{1}<q_{2}$ where $q_{1} \in Q_{1}$ and $q_{2} \in Q_{2}$. For Gorenstein* posets $Q_{1}$ and $Q_{2}$, the poset $Q_{1} * Q_{2}=\left(Q_{1}-\{\hat{1}\}\right)+\left(Q_{2}-\{\hat{0}\}\right)$ is called the join of $Q_{1}$ and $Q_{2}$, and $\Sigma Q_{1}=Q_{1} * B_{2}$, where $B_{2}$ is a Boolean algebra of rank 2, is called the suspension of $Q_{1}$. By [St1, Lemma 1.1], $\Phi_{Q_{1} * Q_{2}}(\mathbf{c}, \mathbf{d})=$ $\Phi_{Q_{1}}(\mathbf{c}, \mathbf{d}) \cdot \Phi_{Q_{2}}(\mathbf{c}, \mathbf{d})$.

Proposition 4.6. Let $P$ be a Gorenstein* poset of rank 5, and let

$$
\Phi_{P}(\mathbf{c}, \mathbf{d})=\mathbf{c}^{4}+\alpha_{\{1\}} \mathbf{d} \mathbf{c}^{2}+\alpha_{\{2\}} \mathbf{c d c}+\alpha_{\{3\}} \mathbf{c}^{2} \mathbf{d}+\alpha_{\{1,3\}} \mathbf{d}^{2}
$$

be its $\mathbf{c d}$-index. Suppose $\alpha_{\{2\}}=0$. Then there are Gorenstein* posets $P_{1}$ and $P_{2}$ of rank 3 such that $P=P_{1} * P_{2}$. In particular, $\alpha_{\{1,3\}}=\alpha_{\{1\}} \alpha_{\{3\}}$.

Proof. Let $r$ denote the rank function $r: P \rightarrow\{0,1, \ldots, 5\}(r(\hat{0})=0, r(\hat{1})=5)$. Let $P_{1}:=\{F \in P: r(F) \leq 2\}$ and $P_{2}:=\{F \in P: r(F) \geq 3\}$.

As $P$ is Gorenstien*, to show that $P=P_{1}+P_{2}$ it is enough to show that $P_{2} \cup\{\hat{0}\}$ is Gorenstien* (as a Gorenstien* poset contains no proper subposet which is Gorenstien* of the same rank, and each interval $[F, \hat{1}]$ with $r(F)=2$ in $P$ is Gorenstien*). For this, it is enough to show that any rank 4 element in $P$ covers exactly two rank 3 elements in $P$. Indeed, this guarantees that the dual poset to $P_{2}$, denoted $P_{2}^{*}$, is the face poset of a union of CW 1-spheres, and as $P$ is Gorenstien* so is its dual $P^{*}$, hence $P_{2}^{*}$ is Cohen-Macaulay since $P_{2}^{*}$ is a rank selected poset [St2, III, Theorem 4.5], which implies that $P_{2}^{*}$ is the face poset of one CW 1-sphere, i.e. $P_{2} \cup\{\hat{0}\}$ is Gorenstien*.

Let $F$ be a rank 4 element of $P$. Then $P$ is a subdivision of $\Sigma([\hat{0}, F])$ (Recalling [EK, Definition 2.6], this is shown by the map $\phi: P \rightarrow \Sigma([\hat{0}, F]), \phi(\sigma)=\sigma$ if $\sigma<F$, $\phi(\sigma)=\sigma_{1}$ if $\sigma$ and $F$ are incomparable, and $\phi(F)=\sigma_{2}$, where $\sigma_{1}, \sigma_{2}$ are the rank 4 elements in $\Sigma([\hat{0}, F]))$. Thus, by Lemma 2.4 , the coefficient of cdc in the cd-index of $\Sigma([\hat{0}, F])$ is zero, hence the coefficient of the monomial $\mathbf{c d}$ in the cd-index of $[\hat{0}, F]$ is zero.

This fact implies, when expanding the cd-index of $[\hat{0}, F]$ in terms of $\mathbf{a}, \mathbf{b}$, that $h_{\{3\}}([\hat{0}, F])$ equals the coefficient of $\mathbf{c}^{3}$, namely $h_{\{3\}}([\hat{0}, F])=1$. Switching to the flag $f$-vector of $[\hat{0}, F]$ we get $f_{\{3\}}([\hat{0}, F])=h_{\emptyset}([\hat{0}, F])+h_{\{3\}}([\hat{0}, F])=1+1=2$. Thus, $F$ covers exactly two rank 3 elements in $P$.

Example 4.7. Consider the 2 -FFK vector $(1,6,7)$. We claim that $\Phi_{P}(1, \mathbf{d}) \neq$ $1+6 \mathbf{d}+7 \mathbf{d}^{2}$ for all Gorenstein* poset $P$ of rank 5 . Indeed, if $\Phi_{P}(1, \mathbf{d})=1+6 \mathbf{d}+7 \mathbf{d}^{2}$, 
then $\alpha_{\{1,3\}}=7$. Then $\alpha_{\{1\}}+\alpha_{\{3\}}=6$ and $\alpha_{\{2\}}=0$ by Proposition 4.4, which contradicts Proposition 4.6.

A similar argument shows that $\left(1,2 a, a^{2}-2\right)$, where $a \geq 3$, is 2 -FFK, but not realizable as the $\mathbf{d}$-polynomial of a Gorenstein* poset of rank 5 .

\section{REFERENCES}

[BK] M.M. Bayer and A. Klapper, A new index for polytopes, Discrete Comput. Geom. 6 (1991), 33-47.

[BE] L.J. Billera and R. Ehrenborg, Monotonicity of the cd-index for polytopes, Math. Z. 233 (2000), 421-441.

[BM] H. Bruggesser and P. Mani, Shellable decompositions of cells and spheres, Math. Scand. 29 (1971), 197-205.

[EK] R. Ehrenborg and K. Karu, Decomposition theorem for the cd-index of Gorenstein posets, J. Algebraic Combin. 26 (2007), 225-251.

[ER] R. Ehrenborg and M. Readdy, Coproducts and the cd-index, J. Algebraic Combin. 8 (1998), 273-299.

[FFK] P. Frankl, Z. Füredi and G. Kalai, Shadows of colored complexes, Math. Scand. 63 (1998), 169-178.

[Ga] Ś.R. Gal, Real root conjecture fails for five- and higher-dimensional spheres, Discrete Comput. Geom. 34 (2005), 269-284.

[Ka] K. Karu, The cd-index of fans and posets, Compos. Math. 142 (2006), 701-718.

[NP] E. Nevo and T.K. Petersen, On $\gamma$-vectors satisfying the Kruskal-Katona inequalities, Discrete Comput. Geom. 45 (2010), 503-521.

[NPT] E. Nevo, T.K. Petersen and B.E. Tenner, The $\gamma$-vector of a barycentric subdivision, $J$. Combin. Theory Ser. A 118 (2011), 1364-1380.

[St1] R.P. Stanley, Flag $f$-vectors and the $c d$-index, Math. Z. 216 (1994), 483-499.

[St2] R.P. Stanley, Combinatorics and commutative algebra, Second edition, Progr. Math., vol. 41, Birkhäuser, Boston, 1996.

Satoshi Murai, Department of Mathematical Science, Faculty of Science, YamAguchi University, 1677-1 Yoshida, YAmaguchi 753-8512, JAPAN

E-mail address: murai@yamaguchi-u.ac.jp

Department of Mathematics, Ben Gurion University of the Negev, Be'er Sheva 84105, ISRAEL

E-mail address: nevoe@math.bgu.ac.il 